\title{
The role of nontuberculous mycobacteria in the diagnosis, management and quantifying risks of tuberculosis in Tanga, Tanzania
}

\author{
ABUBAKAR S. HOZA ${ }^{1,2^{*}}$, ATHUMANI M. LUPINDU ${ }^{3}$, SAYOKI G.M. MFINANGA ${ }^{4}$, IRMGARD MOSER ${ }^{5}$ and \\ BRIGITTE KÖNIG \\ ${ }^{1}$ Department of Medical Microbiology and Epidemiology of Infectious Diseases, Medical Faculty, University of \\ Leipzig, Germany \\ ${ }^{2}$ Department of Veterinary Microbiology \& Parasitology, Faculty of Veterinary Medicine, Sokoine University of \\ Agriculture, Morogoro, Tanzania \\ ${ }^{3}$ Department of Veterinary Medicine \& Public Health, Faculty of Veterinary Medicine, Sokoine University of \\ Agriculture, Morogoro, Tanzania \\ ${ }^{4}$ National Institute for Medical Research, Muhimbili Research Centre, Dar es Salaam-Tanzania \\ ${ }^{5}$ Friedrich Loeffler Institut, Institute of Molecular Pathogenesis, Naumburger Str. 96a, 07743 Jena, Germany
}

\begin{abstract}
Background: The role of nontuberculous mycobacteria (NTM) in tuberculosis (TB) diagnosis is well documented in many developing settings. However, this has not been the case in many resource poor settings like Tanzania. This study aimed at understanding the role of NTM in the diagnosis and management of TB in resource poor settings of Tanzania.

Methods: A cross-sectional study was conducted in Tanga, Tanzania. Patients with symptoms suggestive of TB self-referred to health care facilities were recruited. Two sputum samples were collected for standard direct smear microscopy. Culture was performed using BacT/Alert 3D system, LöwensteinJensen and Gottsacker slopes. Identification of Mycobacterium tuberculosis and NTM was done by using GenoType ${ }^{\circledR} \mathrm{MTBC}$ and GenoType ${ }^{\circledR} \mathrm{CM} / \mathrm{AS}$, respectively.

Results: A total of 372 patients were involved in the study. Eighty-one (21.8\%) patients were diagnosed as having $M$. tuberculosis by the isolation of the organism from cultures of sputum. Further analysis of culture showed that $8.1 \%$ (30/372) were NTM with $7 / 372$ (1.9\%) cases of NTM classified as pulmonary tuberculosis (PTB) patients. Ziehl Neelsen stain had a sensitivity of $68.8 \%$ and produced 10 false negative results. On the other hand, Fluorescence stain had a sensitivity of $85.7 \%$ and gave seven false negative samples when compared with culture results. Weight loss $(p=0.0001)$, fatigue $(p=0.003)$, fever $(p=0.038)$ and night sweats $(p=0.004)$, young population $(18-40$ years $)(p=0.0352)$, males $(p=0.0025)$ were important risk factors for TB. Four out of 30 NTM diagnosed by culture received first line anti-TB treatment suggesting that a good proportion of patients $(4 / 65,6.2 \%)$ were mistreated as TB patients.

Conclusion: Inefficient screening of TB patients in resource poor settings and prevalent increase of NTM may contribute to over diagnosis of TB cases. The need to integrate NTM diagnosis in the routine management of TB is urgently needed for designing effective tuberculosis prevention and control strategies in the country.
\end{abstract}

Keywords: tuberculosis, nontuberculous mycobacteria, management, Tanzania

\section{Introduction}

Tuberculosis (TB) caused by Mycobacterium tuberculosis (MTB) complex remains a major public health challenge worldwide and a leading cause of morbidity and mortality throughout subSaharan Africa (WHO, 2009). The emergency of multidrug- resistant (MDR) TB and co-infection with HIV have continued to threaten global TB control, especially in resource poor settings of sub-Saharan Africa (Cobelens et al., 2008; Migliori et al., 2008a,b; WHO, 2010a). The problem in many resource poor settings like Tanzania is further compounded by weak health care systems, and inadequate laboratory infrastructure.

The incidence of all forms of TB in Tanzania is estimated at 295 cases per 100,000 persons per year (NTLP, 2011). The country has achieved commendable landmarks in fighting

\footnotetext{
*Correspondence Email: abshoza@gmail.com.
} 
against TB, and listed among 13 countries from the high-burden countries (HBCs) that achieved treatment success rate target of $85 \%$ set by the World Health Organization for new sputum smear-positive cases of pulmonary TB (WHO, 2010a). Case detection rate for new smear positive TB cases remains low at 51\%, far below the WHO's target of 70\%. The WHO report further shows an alarming trend of increased active TB cases in Tanzania due to HIV/AIDS epidemic, which is associated with 60 percent increase in active TB (WHO, 2010a).

In spite of these achievements, a number of challenges face the TB control programme. The current surveillance system still relies on passive case finding among individuals who self-referred to health facilities, followed by diagnosis based on the presumptive clinical TB symptoms and laboratory diagnosis using insensitive direct sputum smear microscopy (Mfinanga et al., 2007; Matee et al., 2009). Typically, this method has been shown to be poor in performance, with low detection rate of 56 to $68 \%$ resulting in missing of half of the cases (WHO, 2009). The sensitivity is reported to be further reduced to $43-51 \%$ among TB/HIV co-infected patients (Sonnenberg et al., 2001).

Several studies have demonstrated that serious infections with non-tuberculosis mycobacteria (NTM) remain a major public health concern in many TB-endemic countries (Crump et al., 2009; Buijtels et al., 2010; Maiga et al., 2012). NTM can present with clinical and radiologic manifestations comparable to $M$. tuberculosis. Most often, it is not feasible to differentiate between NTM and MTB microscopically in situations where sputum culture and other diagnostic tests are not done. Increased prevalence of nontuberculous mycobacteria (NTM) infections among TB patients which are difficult to differentiate from MTBC further jeopardises the control efforts to contain the disease (Migliori et al., 2008).

It is apparently therefore, that the need for a quick and accurate diagnosis of TB is critically important. Accurate diagnosis of MTB will not only ensure accurate and timely management of TB patients, but also will reduce morbidity, mortality, economic loss, further spreading of infection and emergence of MDR strains (Kox et al., 1994; Moulding et al., 2004). This study therefore aimed at determining the role of nontuberculous mycobacteria (NTM) in diagnosis, treatment and quantifying risks for TB transmission in Tanga, Tanzania.

\section{Materials and Methods}

\section{Study area, design and population}

This cross-sectional study was conducted in Tanga, Tanzania from November 2012 to January 2013. The study involved four health facilities, namely Makorora and Ngamiani health centres and Bombo Regional Referral hospital in Tanga City and the Designated District Hospital in the nearby Muheza district. All patients with clinical signs and symptoms suggestive of TB self-referred to four primary health care facilities were eligible for the study. Demographic information and data were collected only after provision of informed consent. A careful cross examination of patient history for previous anti-TB treatment using a structured questionnaire was used to classify patients as 'new' or 'previous treated' TB cases. A patient was considered as "new case" if had no prior anti-TB treatment for more than 1 month and was considered 'previous treated' if had received anti-TB treatment lasting at least 1 month (Abdel-Aziz, 2003). There were no restrictions on inclusion criteria regarding clinical symptoms and age of the patients.

\section{Sputum and data collection}

Demographic data were obtained using structured questionnaire administered to the patients attending the health care facilities who provided informed consent to the study. Two sputum samples (one spot during the initial visit to the clinic and one early morning) were collected into small autoclavable wide mouth glass bottles. The specimens were examined using the standard direct smear microscopy at the respective facilities using either Ziehl Neelsen (ZN) stain (Makorora and Ngamiani health centres) or fluorescence stain (Bombo and Muheza). Diagnosis of smear- 
positive TB was performed based on the National Tuberculosis and Leprosy Programme Guidelines (NTLP, 2006). All morning sputum samples kept at $-20^{\circ} \mathrm{C}$ at the respective clinics. No preservatives were added until shipped to the mycobacteriology laboratory at the Institute of Medical Microbiology and Epidemiology of Infectious Diseases, University Hospital, Leipzig, Germany for culture and molecular analysis. HIV status of the patients was determined by rapid HIV screening method at the treatment and care centres (CTC) of the respective clinics.

\section{Sputum culture and identification of mycobacterial isolates}

Sputum specimens were digested and decontaminated using $\mathrm{N}$-acetyl-I-cysteine-sodium hydroxide method (Kent \& Kubica, 1985) and were re-examined for the presence of acid-fast bacilli by fluorescence stain in Leipzig. The isolates were cultured in BacT/Alert 3D liquid culture system (bioMe'rieux, Mercy-l' Ėtoile, France) and on Löwenstein-Jensen and Gottsacker slants (ArteltENCLIT GmbH, Wyhra, Germany). Gottsacker slopes contains sodium pyruvate for isolation of $M$. bovis. Cultures were incubated at $37^{\circ} \mathrm{C}$ for up to 8 weeks. Confirmation of $M$. tuberculosis and NTM was done based on presumptive phenotypic appearance of colonies and by line probe assay GenoType ${ }^{\circledast}$ MTBC for identification of $M$. tuberculosis and GenoType ${ }^{\circledR} \mathrm{CM} / \mathrm{AS}$ for the identification of common and additional NTMs. The assay kits were obtained from Hain Lifescience, Nehren, Germany.

\section{Data analysis}

Descriptive statistics such as frequencies, means and proportions for different study attributes were computed by using Open Epi software version 3. A 2x2 contingency table for comparison of direct smear microscopy and the gold standard cultural method was built and prevalence, sensitivity, specificity and kappa statistic of agreement were calculated by using SAS software version 9. The Chi-square test of association between TB status of study participants and clinical signs were computed at significant level of $5 \%$. The risk factors associated with positive TB status were quantified by logistic regression using SAS software version 9. The model building process followed a backward stepwise strategy whereby univariable analysis of all variables preceded the multivariable analysis. The variables included age, gender, occupation, previous TB history, smoking, diabetes and HIV/AIDS status. A variable qualified for multivariable analysis when it had a p-value of less than 0.25 . A multivariable model was run and an iterative process of removing a variable with highest $p$-value was done until all variables in the model had $p$-value of less than 0.05 . A variable was regarded as a confounder and retained in the final model when its removal from the multivariable model caused a more than $25 \%$ or between -0.4 and 0.4 changes in coefficient of any other variable. Model fitness test was assessed by Hosmer-Lemeshow test.

\section{Ethical considerations}

The protocol for this study was approved by the Medical Research Coordinating Committee of National Institute for Medical Research, Tanzania (Certificate No. NIMR/HQ/R.8a/Vol. IX/1401). Written informed consents were obtained from all participants of this study. For illiterate participants, there was an independent witness present during the consenting process, who then signed the relevant witness section of the consent form.

\section{Results}

\section{Comparison of results of staining techniques and culture}

A total of 372 TB suspects including 312 new and 60 previously treated cases were recruited. Eightyone of the $372(21.8 \%)$ patients were diagnosed as having $M$. tuberculosis by the isolation of the organism from cultures of sputum. A total of 176 samples were analysed by ZN staining while 196 samples were analysed by fluorescence staining. $Z N$ stain was able to detect 22 out of 32 positive samples by culture technique. This means ZN method had a sensitivity of (68.8\%) and produced 10 
false negative results. On the other hand, Fluorescence staining was able to detect 42 out of 49 positive samples by culture technique (sensitivity $=85.7 \%$ ) and seven $(7)$ samples were false negative (Table 1). Moreover, culture results showed that $8.1 \%$ (30/372) of the sputum was positive for NTM, with $7 / 372(1.9 \%)$ of culture confirmed NTM recorded as TB positive case by the health facilities. The remaining 68.3\% (254/372) were culture negative for $M$. tuberculosis at 8 weeks.

Table 1: Relationship of results between ZN stain, Fluorescence stain and culture $(n=372)$

\begin{tabular}{|c|c|c|c|c|c|}
\hline & & & \multicolumn{3}{|l|}{ Culture } \\
\hline & & & positive & negative & Total \\
\hline \multirow[t]{5}{*}{ Stain } & ZN & Positive & 22 & 0 & 22 \\
\hline & & Negative & 10 & 144 & 154 \\
\hline & Fluorescence & Positive & 42 & 0 & 42 \\
\hline & & Negative & 07 & 147 & 154 \\
\hline & & Total & 81 & 291 & 372 \\
\hline
\end{tabular}

\section{Assessment of criteria for suspecting TB cases in Tanga}

Currently, one of the defining criteria for suspecting TB is cough for over 2 weeks. In attempt to establish how correctly the clinics were able to assess the patients presented with TB cardinal symptoms and the actual TB cases, we analysed the association of TB status with the clinical manifestations presented during patients visit to the TB facilities. Results show that weight loss ( $\mathrm{p}$ $=0.0001)$, fatigue $(p=0.003)$, fever $(p=0.038)$ and night sweats $(p=0.004)$ were statistically significantly associated with TB positive status of patient in Tanga (Table 2).

Table 2: Assessment of TB status and clinical signs presented by patients in Tanga $(n=372)$

\begin{tabular}{llllllll}
\hline Variable & Category & $\mathbf{n}$ & TB + & TB - & Df & $\mathbf{X}^{2}$ value & p-value \\
\hline Cough $\geq 2$ weeks & Yes & 327 & 75 & 252 & 1 & 2.14 & 0.14 \\
Bloody sputum & No & 45 & 6 & 39 & & & \\
Night sweats & Yes & 44 & 11 & 33 & 1 & 0.3 & 0.58 \\
No & Yes & 249 & 70 & 258 & & & \\
Fatigue & No & 123 & 16 & 107 & & & 0.004 \\
& Yes & 208 & 57 & 151 & 1 & 8.78 & 0.003 \\
Fever & No & 164 & 24 & 140 & & & \\
& Yes & 254 & 63 & 191 & 1 & 4.31 & 0.038 \\
Dyspnoea & No & 118 & 18 & 100 & & & \\
Yes & 176 & 46 & 130 & 1 & 4.04 & 0.045 \\
Weight loss & No & 194 & 34 & 160 & & & \\
& Yes & 138 & 49 & 89 & 1 & 24.16 & 0.0001 \\
\hline
\end{tabular}

\section{Quantification of risk factors for occurrence Tuberculosis in patients in Tanga}

Univariable analysis of TB status (dependent variable) against age, gender, occupation, TB history, smoking, diabetes, HIV/AIDS (independent variables) was run. Results of this analysis revealed that age, sex and occupation were significantly associated with positive TB status in patients in study area (Table 3). 
Table 3. Single and modelled multiple risk factors for occurrence of TB

\begin{tabular}{|c|c|c|c|c|c|c|}
\hline Risk factor & Response & $\mathrm{n}(\%)$ & $\begin{array}{l}\text { TB positive } \\
\mathrm{n}(\%)\end{array}$ & OR & $95 \% \mathrm{Cl}$ & P value \\
\hline \multirow[t]{4}{*}{ Age (years) } & $5-17$ & 16 & $4(4 \cdot 9)$ & 0.211 & $0.046-0.962$ & 0.3637 \\
\hline & $18-40$ & 184 & $49(60.5)$ & 0.193 & $0.067-0.561$ & 0.0352 \\
\hline & $41-60$ & 111 & $24(29.6)$ & 0.254 & $0.084-0.772$ & 0.3896 \\
\hline & $>60$ & 61 & $4(4 \cdot 9)$ & & & \\
\hline \multirow[t]{2}{*}{ Sex } & Male & $192(51.6)$ & $54(66.7)$ & 2.217 & $1.323-3.715$ & 0.0025 \\
\hline & Female & $180(48.4)$ & $27(33 \cdot 3)$ & & & \\
\hline \multirow[t]{2}{*}{ Occupation } & High contact & $147(39.5)$ & $46(56.8)$ & 2.472 & $1.497-4.083$ & 0.0004 \\
\hline & Low contact & $225(60.5)$ & $35(43.2)$ & & & \\
\hline \multirow[t]{2}{*}{ Previous TB history } & Yes & 61 & $11(13.6)$ & 0.757 & $0.374-1.533$ & 0.4399 \\
\hline & No & 311 & $70(86.4)$ & & & \\
\hline \multirow[t]{2}{*}{ Smoking } & Yes & 79 & $22(27.5)$ & 1.558 & $0.881-2.753$ & 0.1273 \\
\hline & No & 293 & $58(72.5)$ & & & \\
\hline \multirow[t]{2}{*}{ Diabetes } & Yes & 2 & $1(1.2)$ & 3.613 & $0.223-58.396$ & 0.3657 \\
\hline & No & 370 & $80(98.8)$ & & & \\
\hline \multirow[t]{3}{*}{ HIV/AIDS status } & Positive & 57 & $7(8.6)$ & 2.235 & $0.954-5.235$ & 0.0682 \\
\hline & Negative & 100 & $23(28.4)$ & 1.047 & $0.597-1.837$ & 0.2691 \\
\hline & Unknown & 215 & $51(63)$ & & & \\
\hline \multicolumn{7}{|c|}{ Final Model Multiple risk† } \\
\hline \multirow[t]{2}{*}{ Sex } & Male & $192(51.6)$ & $54(28.13)$ & 1.909 & $1.124-3.244$ & 0.0167 \\
\hline & Female & $180(48.4)$ & $27(15)$ & & & \\
\hline \multirow[t]{2}{*}{ Occupation } & High contact & $147(39.5)$ & $46(31.3)$ & 2.188 & $1.31-3.654$ & 0.0028 \\
\hline & Low contact & $225(60.5)$ & $35(15.6)$ & & & \\
\hline
\end{tabular}

$\dagger$ The multivariable analysis resulted into a final model with two variables, namely gender and occupation. Male patients had 1.9 times higher risk of suffering TB while people involved in high contact activities had 2.188 times odds of getting TB. This model well fitted the variables as shown by Hosmer-Lemeshow test with $\mathrm{X}^{2}=0.0092, \mathrm{df}=2$ and $\mathrm{p}$-value of 0.99 .

\section{Comparison of diagnostic results and treatment}

We evaluated the treatment of different subjects based on smear microscopy results and culture results. Among the 65 subjects receiving first line anti-TB drugs, 25 (38.5\%) were diagnosed by ZN staining and $40(61.5 \%)$ by Fluorescence staining. Moreover, 64 subjects whose samples were processed by $\mathrm{ZN}$ staining and 47 subjects by fluorescence staining were empirically treated with broad spectrum antibiotics. Out of 196 subjects who did not receive any medication, 87 and 109 subjects had their samples processed by ZN and fluorescence staining, respectively (Table 4).

Table 4. Distribution of treatment type by staining technique $(n=372)$

\begin{tabular}{|c|c|c|c|c|}
\hline & & \multicolumn{3}{|c|}{ Smear technique } \\
\hline & & ZN & Fluorescence & Total \\
\hline \multirow[t]{4}{*}{ Treatment } & $1^{\text {st }}$ line anti-TB drugs & 25 & 40 & 65 \\
\hline & Broad spectrum antibiotics & 64 & 47 & 111 \\
\hline & No treatment & 87 & 109 & 196 \\
\hline & Total & 176 & 196 & 372 \\
\hline
\end{tabular}

Of the 81 patients diagnosed as TB positive by culture, 54 were already on first line anti-TB drugs, while 11 were empirically treated with broad spectrum antibiotics and 16 were not given any medication following smear microscopy results. Out of 291 TB negative subjects, 11 were given first line anti-TB drugs, 100 were given broad spectrum antibiotics and 180 received no therapy (Table 5). 
Table 5. Comparison of diagnostic results and treatment for the study subjects $(n=372)$

\begin{tabular}{lllll}
\hline & & \multicolumn{3}{l}{ Culture results } \\
\cline { 3 - 5 } & & Positive & Negative & Total \\
\hline Treatment & First line anti-TB & 54 & 11 & 65 \\
& Broad spectrum antibiotics & 11 & 100 & 111 \\
& No treatment & 16 & 180 & 196 \\
\hline & Total & 81 & 291 & 372 \\
\hline
\end{tabular}

Further analysis indicated that subjects diagnosed as NTM positive by culture had already received different medication following smear microscopy results (Table 6 ). Four subjects received first line anti-TB drugs, 12 received broad spectrum antibiotics and 14 received no medication.

Table 6. Association of NTM diagnosis and treatment for the study participants $(n=372)$

\begin{tabular}{lllll}
\hline & & \multicolumn{3}{l}{ NTM diagnosis } \\
\cline { 3 - 5 } & & Positive & Negative & Total \\
\hline Treatment & $1^{\text {st }}$ line anti-TB & 4 & 61 & 65 \\
& Broad spectrum antibiotics & 12 & 99 & 111 \\
& No medication & 14 & 196 & 196 \\
& Total & 30 & 342 & 372 \\
\hline
\end{tabular}

\section{Discussion}

Sputum smear microscopy is the mainstay for pulmonary tuberculosis screening in high-burden countries (WHO, 2011). The method has proven to be rapid and easily affordable means of diagnosing PTB in many resource poor settings, where TB is still a major public health burden. Findings from this study showed that the sensitivity of ZN method was $68.8 \%$ and that of Fluorescence staining was $85.7 \%$ when compared to culture results. This finding is not surprising since ZN stain is known to have a low level of sensitivity compared to the Fluorescence method (Ulukanligil et al., 2000). Finding that about one-fifth of the patients were diagnosed with $M$. tuberculosis by the isolation of the organism from cultures of sputum suggest that a significant proportion of the patients had sputum culture positive for $M$. tuberculosis. These findings reaffirms the consolidate the findings that the performance characteristics of smear microscopy is poor in the diagnosis of PTB (WHO, 2009). In patients co-infected with HIV the performance has shown to be further lower (Sonnenberg et al., 2001).

Generally, smear-negative PTB accounts for $30 \%$ of PTB cases per annum in developing countries (Scherer et al., 2007). The findings suggest that, while from the public health point of view smear-positive cases are considered more significant in diagnosing PTB, smear-negative patients on the other hand may be actual cases of TB as proved at some cases by culture (Tadesse et al., 2011). Since smear microscopy does not only play a pivotal role in the initial diagnosis of PTB but also in monitoring of anti-TB treatment, false negatives can continue to be infection source while false positives may be subjected to unnecessary anti-TB medication. Our results show that over half of the patients diagnosed by Fluorescence method received anti-TB treatment compared to about a third when ZN method was used. In general, passive case finding strategy combined with sub-optimal diagnostic procedures at diagnostic clinics may pose a serious problem in underestimating the burden of the disease.

The diagnostic value of smear microscopy in general is reportedly being eroded not only by increased cases of HIV-related smear negative PTB, but also due to the influence of NTM (Lalit, 2001; Valadas et al., 2003). The finding that a few of the patients had culture confirmed NTM elucidates an important public health and diagnostic challenge of continuing relying on smear microscopy for diagnosis of PTB in settings where dual infections are on the increase. Furthermore, classification of a few of culture confirmed NTM as PTB patients based on the smear microscopy results means that, such patients were managed as individuals with PTB. 
NTM can present with clinical and radiologic manifestations comparable to M. tuberculosis. Since it is not feasible to differentiate between NTM and MTB microscopically in situations where sputum culture and other identification tests are not done, it could imply therefore, that NTM cases may have been treated as PTB for long periods of time. Essentially, NTM treatment differ from PTB treatment. Thus, such patients may show unfavourable response to anti-TB therapy. Additionally, since NTM isolates are often resistant to first-line anti-TB drugs, it may result into classifying and treating these individuals as MDR-TB cases (Tabarsi et al., 2009), that may require the use of second-line drugs which are more toxic and expensive, consequently adding up to waste of resources due to mismanagement of such patients. Several studies have demonstrated that serious infections with NTM remain a major public health concern in many TB-endemic countries (Crump et al., 2009; Buijtels et al., 2010; Maiga et al., 2012).

The presumptive diagnosis of PTB has in practice been based on an algorithm of clinical and radiological (where available) criteria coupled with smear microscopy. Based on this fact, we assessed the defining criteria in predicting PTB positivity at the primary care facilities. Our results showed that, weight loss, fatigue, fever and night sweats were the most likely criteria for TB positivity among patients in Tanga. Since one of the defining criteria for suspecting TB is cough for $\geq 2$ weeks, it can be argued that, this generalization might be contributing to missing a reasonable number of TB cases. This is because patients reporting at the primary diagnostic facilities with cough of less than two weeks are likely to have not been screened for TB under routine programme (Ngadaya et al., 2009). Conversely, experience from the study shows that, a tendency of generalizing all complaints of cough for $\geq 2$ weeks as a gold standard for PTB suspect leads not only to unnecessary increase of workload to already overwhelmed clinics, but also to unrealistic case detection. Inclusion of all patients with cough of more than two weeks may include patients with simple chest infections that are sometimes complicated with cough (Ngadaya et al., 2009; Swai et al., 2011). Furthermore, it may also contribute to increased negative results, delayed reporting and ultimately eroding patient's faith in the services of the laboratory and of the general health system (Wandwalo \& Mørkve, 2000).

Results of risk factors quantification indicated that age, sex and occupation were the important risk factors associated with TB positivity. Young population at the age between 18 to 40 years and males were at most risk of TB infection. Similar trend was also reported during the 2012 prevalence survey (NTLP, 2011). These findings suggest that the patient population in this setting is marked with active transmission; hence, TB is largely seen in younger population. Furthermore, "high contact" subjects like students, community gatherings and other crowd related human activities (including public commute transports) remain at high risk of contracting TB.

In conclusion, unrealistic TB case detection may create a cluster of infectious cases in the community that maintain active TB transmission. Furthermore, classification of $1.9 \%$ of culture confirmed NTM as TB patients at the primary health care facilities suggest that, a substantial number of patients may have been wrongly managed as PTB cases. An inefficient screening plus sub-optimal diagnostic procedures at the primary health care facilities may lead to unrealistic detection of TB cases. There is an urgent need to integrate NTM diagnosis in management of TB for designing effective tuberculosis prevention and control strategies in the country. Promoting of favourable health seeking behaviours and education especially among males, younger population and those at high risk such as crowded human activities need to be strengthened.

\section{Acknowledgements}

We are grateful to the management and technical personnel, of the TB clinics as well as the TB Coordinators in Muheza and Tanga. We thank Ms. Elizabeth Kraftschek from the Department of Medical Microbiology and Epidemiology of Infectious Diseases, University of Leipzig for her technical support. 


\section{Competing interests}

All authors declare that they have no competing interests.

\section{References}

Abdel-Aziz, M. (2003) Guidelines for Surveillance of Drug Resistance in Tuberculosis. 2nd. ed. World Health Organization, Geneva.

Buijtels, P.C.A.M., Van Der Sande, M.A.B., Parkinson, S., Verbrugh, H.A., Petit, P. L. C. \& van Soolingen, D. (2010) Isolation of non-tuberculous mycobacteria at three rural settings in Zambia; a pilot study. Clinical Microbiology and Infection 16, 1142-1148.

Cobelens, F.G.J., Heldal, E., Kimerling, M.E., Mitnick, C.D., Podewils, L.J., Ramachandran, R., Rieder, H.L., Weyer, K. \& Zignol, M. (2008) Scaling up programmatic management of drug-resistant tuberculosis: a prioritized research agenda. PLoS Medicine 5 (7), 1037-1042.

Crump, J.A., van Ingen, J., Morrissey, A.B., Boeree, M.J., Mavura, D.R., Swai, B., Thielman, N.M., Bartlett, J.A., Grossman, H., Maro, V.P. \& Van Soolingen, D. (2009) Invasive disease caused by nontuberculous Mycobacteria, Tanzania. Emerging Infectious Diseases 15, 53-55.

Kent, P.T. \& Kubica, G.P. (1985) Public Health Mycobacteriology: A Guide for the LEVEL III LABORATORY. Atlanta, GA, US Department of Health and Human Services, Public Health Service, Centers for Disease Control.

Kox, L.F.F., Rhienthong, D., Miranda, A.M., Udomsantisuk, N., Ellis, K., van Leeuwen, J., van Heusden, S., Kuijper, I.S. \& Kolk, A.H. (1994) A More Reliable PCR for Detection of Mycobacterium tuberculosis in clinical samples. Journal of Clinical Microbiology 32, 672-678.

Lalit, K. (2001) Improving detection of infectious cases. Indian Journal of Tuberculosis 48, 115-116.

Maiga, M., Siddiqui, S., Diallo, S., Diarra, B., Traoré, B., Shea, Y.R., Zelazny, A.M., Bindongo, D.P.P., Goita, D., Kassambara, H., Hammond, A.S., Polis, M.A., Tounkara, A. \& Hozbor, D.F. (2012) Failure to recognize nontuberculous Mycobacteria leads to misdiagnosis of chronic pulmonary tuberculosis. PLoS One 7 (5), e36902.

Matee, M., Mfinanga, S. \& Holm-Hansen, C. (2009) Anti-TB drug resistance levels and patterns among Mycobacterium tuberculosis isolated from newly diagnosed cases of pulmonary tuberculosis in Dar es Salaam, Tanzania. APMIS 117, 263-267.

Mfinanga, G., Ngadaya, E., Mtandu, R., Mutayoba, B., Basra, D., Kimaro, G., Chonde, T., Ngowi, P., Mfaume, S., Kilale, A., Egwaga, S. \& Kitua, A. (2007) The quality of sputum smear microscopy diagnosis of pulmonary tuberculosis in Dar es Salaam, Tanzania. Tanzania Health Research Bulletin 9, 164-168.

Migliori, G.B., Matteelli, A., Cirillo, D. \& Pai, M. (2008b) Diagnosis of multidrug-resistant tuberculosis and extensively drug-resistant tuberculosis: Current standards and challenges. Canadian Journal of Infectious Diseases and Medical Microbiology 19, 169-172.

Milgliori, G.B., Centis, R., Lange, C., Matteelli, A., Cirillo, D. (2008a) Tuberculosis, one disease, many faces. Archives of Chest Diseases 69, 2-4.

Moulding, T.S., Le, H.Q., Rikleen, D. \& Davidson, P. (2004) Preventing drug-resistant tuberculosis with a fixed dose combination of isoniazid and rifampin. International Journal of Tuberculosis and Lung Diseases 8, 743-748.

Ngadaya, E.S., Mfinanga, G.S., Wandwalo, E.R. \& Morkve, O. (2009) Detection of pulmonary tuberculosis among patients with cough attending outpatient departments in Dar es Salaam, Tanzania: does duration of cough matter? BMC Health Services Research 9, 112.

NTLP (2006) Manual of the National Tuberculosis and Leprosy Programme in Tanzania, 5th ed. Ministry of Health and Social Welfare, National Tuberculosis and Leprosy Programme, Dar es Salaam, Tanzania, $132 \mathrm{pp}$.

NTLP (2011) National Tuberculosis and Leprosy Programme Annual Report. National TB and Leprosy Programme, Ministry of Health and Social Welfare, Dar es Salaam, Tanzania, 50 pp. 
Scherer, L., Sperhacke, R., Jarczewski, C., Cafrune, P.I., Minghelli, S., Ribeiro, M., Mello, F.C.Q., Ruffino-Netto, A., Rossetti, M.L.R. \& Kritski, A.L. (2007) PCR colorimetric dot-blot assay and clinical pretest probability for diagnosis of pulmonary tuberculosis in smear-negative patients. BMC Public Health 7, 1-10.

Sonnenberg, P., Murray, J., R Glynn, J., Shearer, S., Kambashi, B., Godfrey-Faussett, P. (2001) HIV-1 and recurrence, relapse, and reinfection of tuberculosis after cure: a cohort study in South African mineworkers. Lancet 358, 1687-1693.

Swai, H.F., Mugusi, F.M., Mbwambo, J.K. (2011) Sputum smear negative pulmonary tuberculosis: sensitivity and specificity of diagnostic algorithm. BMC Research Notes 4 (1), 475.

Tabarsi, P., Baghaei, P., Farnia, P., Mansouri, N., Chitsaz, E., Sheikholeslam, F., Marjani, M., Rouhani, N., Mirsaeidi, M., Alipanah, N., Amiri, M., Masjedi, M.R. \& Mansouri, D. (2009) Nontuberculous Mycobacteria among patients who are suspected for multidrug-resistant tuberculosis-need for earlier identification of nontuberculosis mycobacteria. American Journal of the Medical Sciences 37, 182-184.

Tadesse, T., Demissie, M., Berhane, Y., Kebede, Y., Abebe, M. \& Pai, M. (2011) Two-thirds of smearpositive tuberculosis cases in the community were undiagnosed in northwest Ethiopia: Population based cross-sectional study. PLoS One 6 (12), e28258.

Ulukanligil, M., Gonul, A. \& Tasçi, S. (2000) A comperative study on the different staining methods and number of specimens for the detection of acid fast bacilli. Memórias do Instituto Oswaldo Cruz 95, 855-858.

Valadas, E., Hansched, T., Fernandes, M.L. \& Antunes, F. (2003) Smear microscopy to diagnose tuberculosis early and prevent further transmission in a population with a high prevalence of HIV infection. Clinical Microbiology and Infection 9, 1045-1047.

Wandwalo, E.R., Mørkve, O., 2000. Knowledge of disease and treatment among tuberculosis patients in Mwanza, Tanzania. International Journal of Tuberculosis and Lung Diseases 4, 10411046.

WHO (2009) Global Tuberculosis Control: Epidemiology, Strategy, Financing. World health Organization, Geneva, 314 pp.

WHO (2010a) Multidrug and extensively drug-resistant TB (M/XDR-TB): 2010 Global Report on Surveillance and Response. World Health Organization, Geneva.

WHO (2010b) Global Tuberculosis Control: Epidemiology Strategy Financing. World health Organization, Geneva, $217 \mathrm{pp}$.

WHO (2011) Global Tuberculosis control. World Health Organization, Geneva Switzerland, 258 pp. 